\title{
Stress level classification using statistical analysis of skin conductance signal while driving
}

\author{
Maryam Memar ${ }^{1,2} \mathbb{D} \cdot$ Amin Mokaribolhassan $^{2}$
}

Received: 28 April 2020 / Accepted: 30 December 2020 / Published online: 10 January 2021

(C) The Author(s) 2021 OPEN

\begin{abstract}
Conventionally, multiple physiological signals are used in the field of stress realization. Although many studies have applied various methods in feature selection and classification, a desirable performance has not yet been achieved. This paper presents a novel method of stress level classification using physiological signals during the real-world driving task. Exploring the most reliable analysis method on a comprehensive physiological signal for stress realization has been commonly investigated in various studies. To obtain a high accuracy approach, a proper classification method should be applied to the most relevant physiological signal. In this study, we evaluate the feasibility and effectiveness of the analysis of variance (ANOVA) classifier learner on the single Galvanic Skin Response (GSR) signal. Three levels of stress are taken into account and two independent features including rising time and amplitude are extracted. These two features are extracted from foot and hand GSR signals in three different scenarios for the sake of training. The result indicates that the foot amplitude feature of the GSR signal solely is a reliable source of stress classification with an accuracy rate of $95.83 \%$ by applying the ANOVA approach. Accordingly, this methodology can substantially reduce the necessity of resorting to the high number of sensors and the corresponding computational burden associated with signal analysis. Besides, reducing the number of sensors during the measurement procedure would increase drivers' safety by reducing the interference between human and measurement devices. In this study, the real data collected by Picard and his coworkers are used, available in the PHYSIONET database.
\end{abstract}

Keywords Stress recognition · Classification learner · Galvanic skin response · Analysis of variance · Driving task

\section{Introduction}

Car-induced accidents are a consequence of drivers' stress or lack of attention which could be affected by emotional events. Traffic congestion could be directly correlated to drivers' mental health, hence developing a continuous monitoring system to automatically detect drivers' stress is vital to enhance safety. Driving in stressful conditions such as city or freeway is associated with a higher rate of accidents, life-threatening situations, and compromises decision-making skills. The physiological response of the human body to stress causes an increase in heart rate, respiration rate, muscle contraction, sweating, etc.[1]. Employing physical indicators and analyzing physiological representatives are techniques that could be used to detect and classify stress. The physiological signals including GSR, electrocardiogram (ECG), respiratory rate (RR), and electromyography (EMG) could be acquired for the aim of stress level monitoring [2, 3]. An experimental procedure to elicit stress conditions has been designed and proposed by Martinez et al. in [4] Using quantitative

$\triangle$ Maryam Memar, Maryam.memar.elec@gmail.com; Amin Mokaribolhassan, amin.mokari@ieee.org| 'Department of Biomedical Engineering, Islamic Azad University, Tabriz, Iran. ${ }^{2}$ School of Electrical Engineering and Robotics, Queensland University of Technology, Brisbane, Australia. 
analysis and different stress levels are classified based on ECG and GSR signals.

Many studies have been conducted to computationally recognize and classify stress levels effectively [5]. George Rigas et al. introduced a method of stress estimation for drivers based on a dynamic Bayesian network (BN) [6]. Healey and Picard [1] achieved an accuracy of $97.4 \%$ for two levels of high and moderate stress based on extracted data from EMG, RR, ECG, and GSR. This research aimed to identify the stress level using the signal fusion of multiple sensors. However, this method is not suitable for classifying stress into three levels and particularly with considering a single signal. They used the support vector machine (SVM) technique based on electroencephalography (EEG) and ECG to recognize driver's fatigue. Zhai [7] and Angus [8] monitored and recorded three types of physiological signals, namely skin temperature (ST), GSR, and blood volume pulse (BVP), and introduced a novel automated system for stress classification. In addition, they used three types of classification methods such as SVM, Naive Bayes (NB) classifier, and decision tree. Recently, A k-nearestneighbor classifier learner is used in [9] for the stress recognition purpose while driving. Here, parameter-based and trend-based techniques are proposed to generate features from ECG signals. The vector Quantization learning method is used in [10] to distinguish stress from relaxation. According to this paper, the most informative signals belong to the cardiac signals.

\subsection{Related work}

GSR and ECG signals are the two most reliable physiological signals for stress recognition [11, 12]. One of the remarkable points that makes GSR signals a better indicator than ECG signals is that GSR signal could be obtained by two sensors on the hand and foot skin, while the collection of ECG signals requires an additional sensor for the chest $[13,14]$. In a practical application, utilizing fewer sensors for drivers during driving is a significant improvement due to the embarrassment which might occur during driving task [15]. It is mostly preferred to use GSR signal along with other physiological signals to increase the accuracy of the classification; however, in the past few years, some literature has focused on the performance of GSR signal solely to reduce the cost and complexity of the required measurement equipment compared to multiple sensor utilization. The feasibility of using a single physiological signal to classify stress levels has been investigated as a practical alternative in [9]. In this study, the advantages of a single signal compared to the multiple signal approach are comprehensively explored. Tang et. al have studied the effect of continuous stress monitoring using GSR on the pattern classification features and illustrated that the
GSR signal facilitates this procedure [16]. In [17] GSR signal along with blood pressure (BP) are investigated for stress level detection. Liu et al. have applied SVM on automatically selected GSR to classify human emotion with an accuracy of $66.67 \%$. Ogorevc et al. have proved that the GSR signal provides larger peaks and amplitude in response to human emotion [18], and [19]. Also, in [20] it has been recognized that the GSR signal has a better correlation with emotional events during driving tasks compared to other physiological signals.

Table 1 is an overview of several stress-detection studies with multiple physiological signals, classification methods, and achieved accuracy. According to this overview, the novelty of the present study could be concluded from two aspects. First, the GSR signal solely is a reliable source of data to discriminate stress since it has an exceptional performance based on a single feature. Second, using a large number of sensors results in a large number of features which leads to considerable computational burden and time taking processing procedure. In addition, using a single sensor is not only provides a cost-effective approach but also does not limit the driver's performance during the driving task. To develop an efficient and reliable system that precisely detects stress levels in drivers, preprocessing calculations, time and cost should be minimized along drivers' comfort and safety should be noted.

\subsection{Contribution}

The importance of both time and frequency features for successfully classifying cognitive tasks is undeniable and has been mentioned in several studies [26]. In this paper, we propose a method to categorize stress into three levels of low, medium, and high based on a single physiological signal during the driving task. We focus on the extensive comparison of the two most reliable features of hand and foot GSR signals. Accordingly, we demonstrate that an appropriate processing and classification method of GSR signal alone can offer it as a viable solution to discriminate stress in the sense of feasibility and effectiveness. The most efficient feature extraction and preprocessing methods are devised to achieve the highest performance and a considerable number of features are extracted and processed. We use a one-way ANOVA classifier learner since it is theoretically simple and powerful, and it is a common way to perform statistical analysis on experiments that has the capability of classifying more than two groups of datasets. The novelty of this approach in utilizing ANOVA is to employ it as a classification technique to reduce the data dimensionality, computational complexity and time, and improve the classification accuracy. Working with one signal in real-time stress recognition is computationally efficient 
Table 1 Overview of stress classification methods and their accuracies

\begin{tabular}{|c|c|c|c|c|}
\hline \multicolumn{5}{|l|}{ Stress Level Classification } \\
\hline Classification method & Physiological signal data & Accuracy & Disadvantages & Advantages \\
\hline NMC [21] & ECG, EDA, RSP, vehicle dynamic data & $90 \%$ & $\begin{array}{l}\text { Measurement with a large number of } \\
\text { sensors }\end{array}$ & Acceptable accuracy \\
\hline LRNN [14] & $\begin{array}{l}\text { PPG, EDA, derived features for HRV } \\
\text { and RSP }\end{array}$ & $89.23 \%$ & $\begin{array}{l}\text { Measurement with a large number of } \\
\text { sensors }\end{array}$ & Acceptable accuracy \\
\hline LDA [1] & ECG, EMG, EDA, RSP & $97.4 \%$ & $\begin{array}{l}\text { Measurement with a large number of } \\
\text { sensors }\end{array}$ & High accuracy \\
\hline $\begin{array}{l}\text { Hybrid decision tree- } \\
\text { tree augmented NBC } \\
{[22]}\end{array}$ & ECG, EDA, RSP, facial EMG & $71.9 \%$ & $\begin{array}{l}\text { Measurement with a large number of } \\
\text { sensors \& Low accuracy }\end{array}$ & - \\
\hline SVM [6] & ECG, EMG, EDA, RSP & $79.3 \%$ & $\begin{array}{l}\text { Measurement with a large number of } \\
\text { sensors \& Low accuracy }\end{array}$ & - \\
\hline KNN [22] & ECG, EDA, RSP & $88.6 \%$ & $\begin{array}{l}\text { Measurement with a large number of } \\
\text { sensors }\end{array}$ & Acceptable accuracy \\
\hline KNN, SVM, ANN [23] & GSR, HR, BR & $77.25 \%$ & $\begin{array}{l}\text { Measurement with a large number of } \\
\text { sensors \& Low accuracy }\end{array}$ & - \\
\hline ANN [24] & ECG, RSP, GSR & $96 \%$ & $\begin{array}{l}\text { Measurement with a large number of } \\
\text { sensors }\end{array}$ & High accuracy \\
\hline Logistic regression [25] & $\mathrm{HR}, \mathrm{RSP}, \mathrm{GSR}$ & $91.4 \%$ & $\begin{array}{l}\text { Measurement with a large number of } \\
\text { sensors }\end{array}$ & High accuracy \\
\hline ANOVA [This study] & GSR & $95.83 \%$ & - & $\begin{array}{l}\text { Measurement with } \\
\text { one sensor \& High } \\
\text { accuracy }\end{array}$ \\
\hline
\end{tabular}

and cost-effective. Moreover, it requires fewer monitoring sensors which makes data acquisition easier and it does not interfere with drivers' natural behavior. Consequently, a recognition rate of $95.83 \%$ using the 'foot' amplitude feature is achieved, where potentially offers a promising solution for future automatic stress detection devices.

This paper is organized as follows: Sect. 2, presents the methodology including data selection, feature extraction, data normalization, and classification method. Section 3 demonstrates the results and discussion. And the final section presents the conclusion of this study.

\section{Methodology}

In order to conduct the proposed methodology, the GSR data MIT-BIH database[1] is employed in this paper. This database is collected by Healey and Picard during a realworld driving experiment. It consists of seventeen drivers' raw data for ECG, EMG, 'foot' and 'hand' GSR, heart rate $(H R)$, marker, and $R R$ and acquired from various wearable sensors. In [1], the experiment was conducted in the Greater Boston area starting from the rest status and after passing three city districts and two highways they returned to the initial location.

\subsection{Data selection}

To recognize stress based on the GSR signal, we have to monitor all related datasets comprehensively. Due to a large number of drivers' dataset, all provided results in this paper belongs to driver 5 as training data. Since some drivers' data sources lack some information, the authors have decided to choose driver 5 to driver 12 . These drivers' data include both foot and hand GSR signals along with a marker. The objective of providing a marker signal which is collected by an observer who accompanied the drivers during the driving task is to support the main signal with alternative data and as an identification tool to define each segment of the experiment. The test duration is $5055 \mathrm{~s}$ and the sampling interval is $30 \mathrm{~ms}$. The overall number of samples is 167183 for each type of signal. The signal segmentation using hand and foot GSR signal and the marker data is presented in Fig. 1. Once the signal is extracted, it is divided into seven segments for the initial and final rest, three city districts, and two highways as data samples. The segmentation of the foot GSR signal regarding the different stress levels is shown in Fig. 2. Each segment is an indicator of low, medium, or high-stress levels. 

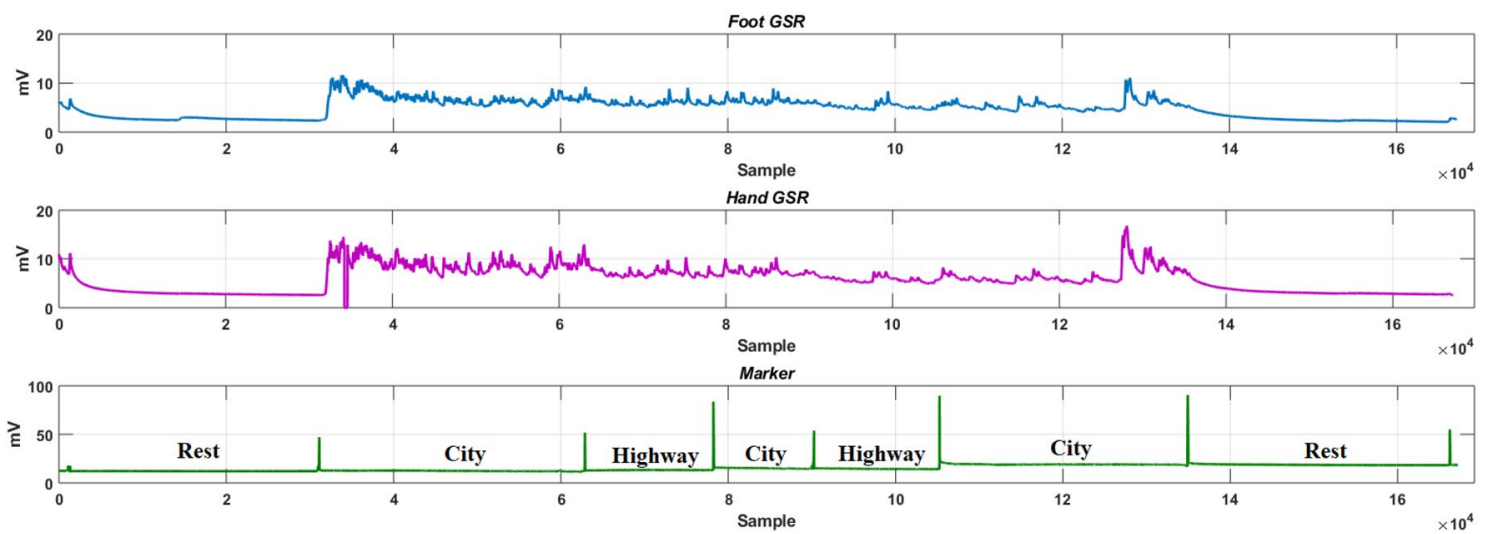

Fig. 1 Signal segmentation for Driver 5 with respect to the marker
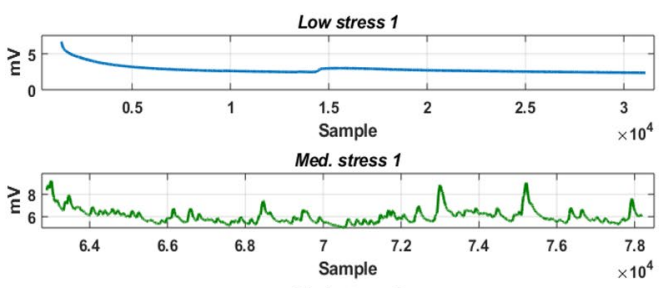

Med. stress 2
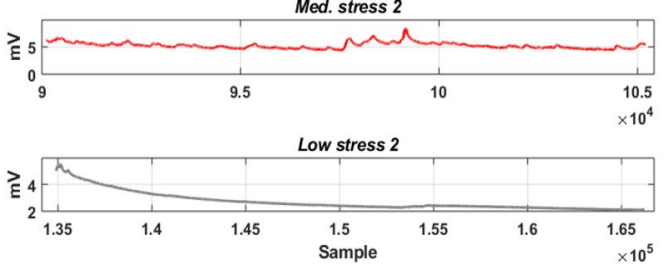

Fig. 2 Stress level segmentation of Driver 5 based on foot GSR Signal

\subsection{Feature extraction}

Two main components of the GSR signal are skin conductance level (SCL) known as tonic level and skin conductance response (SCR) known as a phasic response. Tonic level changes are slow (within ten seconds to $1 \mathrm{~min}$ ) and dependent on the skin condition which could be dry or moist. Hence, SCL is not informative enough for this research objective. While SCR rides on top of the tonic level with rapid fluctuations, it is easy to find the peaks and bursts which could provide us with valuable information to identify the emotional stimulus events. Generally, the duration of the stimulus event is approximately 1 to $5 \mathrm{~s}$ after the onset of the emotional stimulus [27].

According to Fig. 3, four features could be utilized to characterize SCR. These features are introduced as follows:

(1) Latency of response onset (SCR lat.):
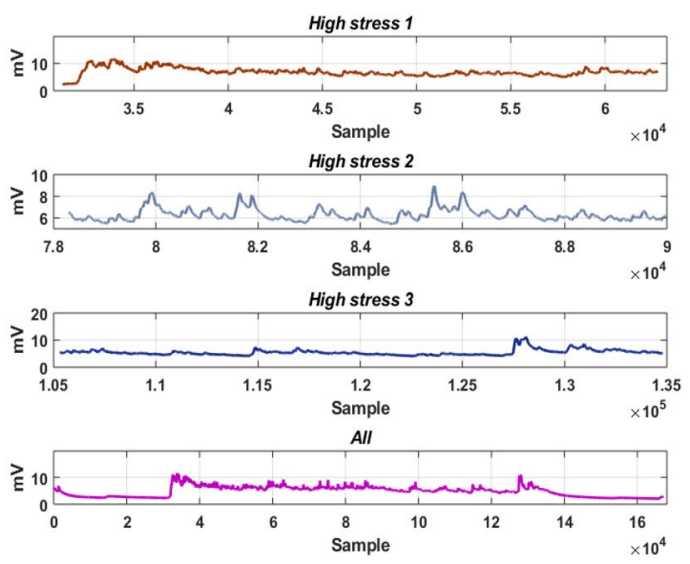

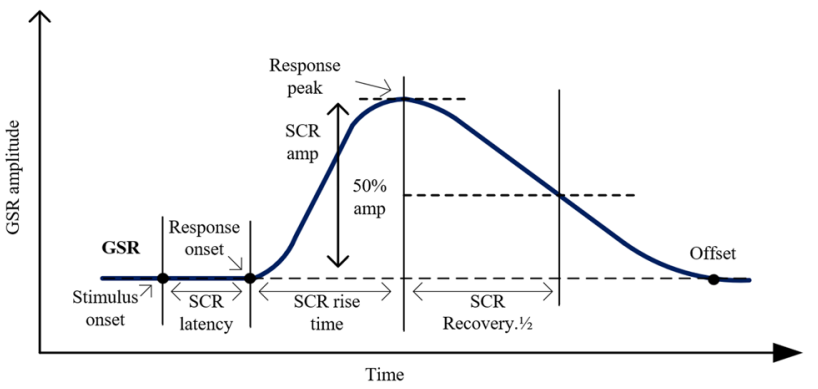

Fig. 3 Characteristics of the SCR in GSR signal

Generally, it may take 1 to $5 \mathrm{~s}$ after the stimulus onset to reach the minimum amplitude. The response onset particularly sets to the time that the SCR experiences the minimum amplitude. Here, SCR changes are disregarded before this period and they would be considered as non-specific skin conductance responses that happen 
spontaneously in the body every 1 to $3 \mathrm{~min}$ and are not considered as a stimulus.

(2) The amplitude (SCR amp):

In this paper, we find the difference in the peak amount and obtain minimum amplitude for each stress segment.

(3) Rise time (SCR rise time):

This feature indicates the time of rising from the response onset to the response peak.

(4) Half time value of the recovery time (SCR recovery $1 / 2)$ :

Here, the duration from the peak to the $50 \%$ recovery is needed to be found.

\subsection{Data normalization and segmentation}

Since noise is an inevitable part of the signal in collecting GSR signal, the selection of filter and associated cut-off frequency is vital to make the GSR signal smooth and obtain the SCR response and remove the SCL. Therefore, an appropriate fourth-order low pass elliptical filter, as shown in Fig. 4, is designed with $0.1 \mathrm{~dB}$ maximum ripple in the pass-band, $40 \mathrm{~dB}$ drop in the stop-band, and $4 \mathrm{~Hz}$ cut-off frequency which is applied to both 'foot' and 'hand' GSR data to remove the noise of high frequency.

The signals are downsampled to obtain particular values at specific time intervals. Since skin conductance variation in different individuals is inescapable, the signals are required to be normalized. For this purpose, the one-step difference signal is devised to eliminate possible effects. It is worthwhile to mention that these procedures are applied to each segment of each signal thoroughly. The first-order difference equation is used to normalize the signal of foot GSR for medium stress as

$Y=[X(2)-X(1), X(3)-X(2), \ldots, X(m)-X(m-1)]$.

where $Y$ is the output signal after implementing the firstorder difference equation, $X$ is the raw signal and $m$ shows the number of samples in each dataset. The processed data is shown in Fig. 5.

Fig. 4 Designed elliptical filter
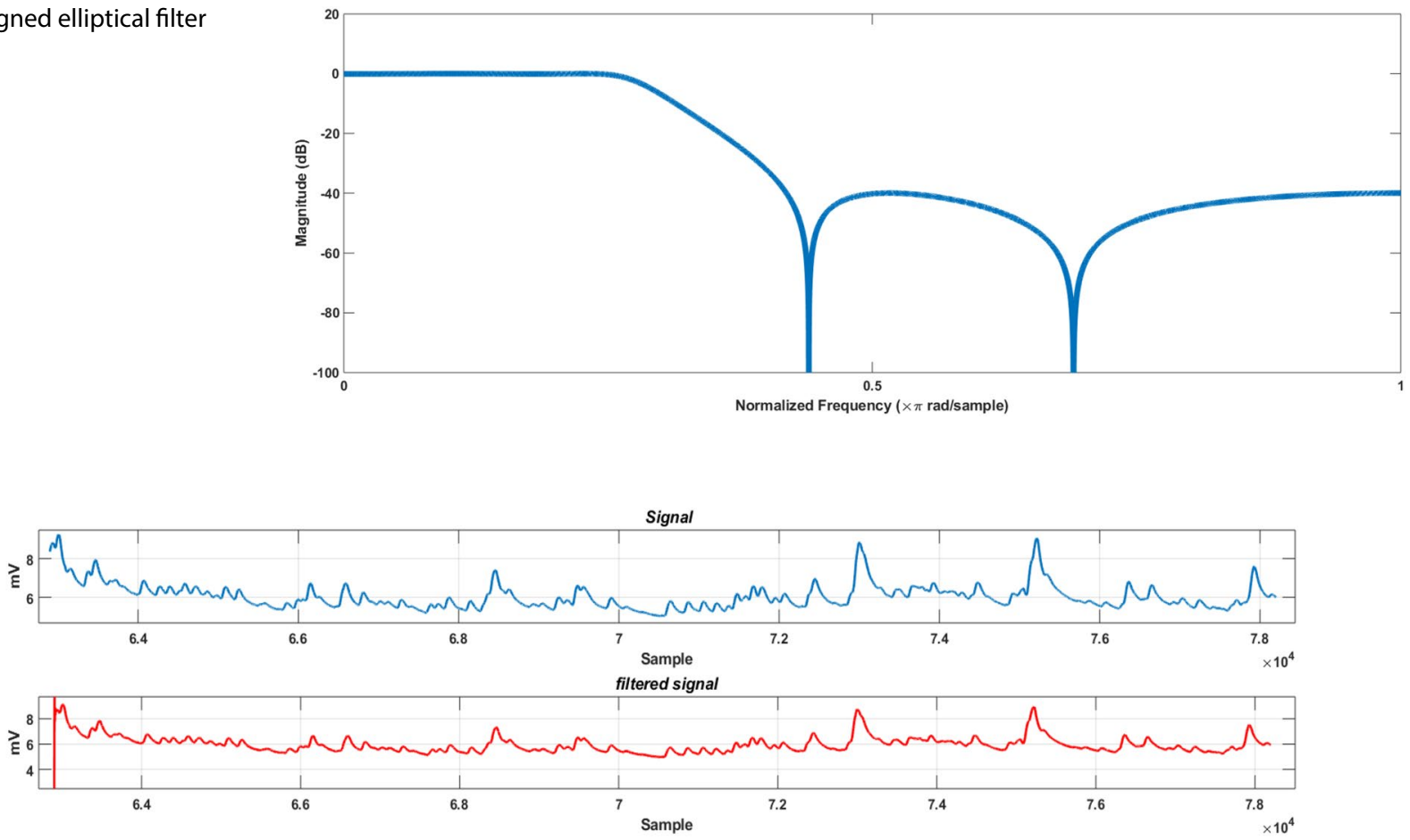

1st differnce of signal

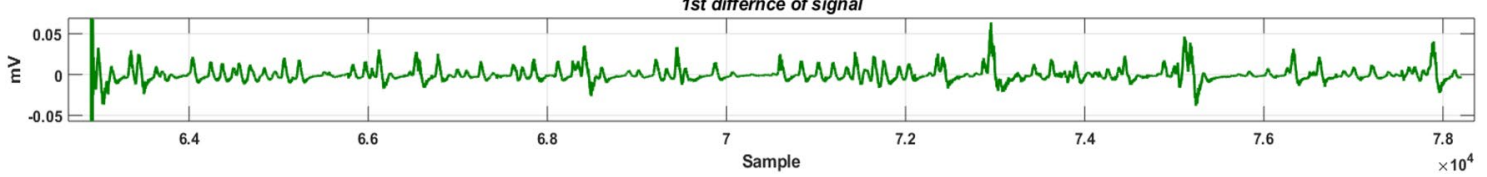

Fig. 5 Original, filtered, and first-order difference signals of driver 5 (Medium level segment) 
The derived first-order difference signal represents the GSR phasic response and associated stimulus point. The stimulus point is identified by finding the significant start point considering amplitudes higher than $0.001 \mathrm{mV}$ to remove the tonic level. In the next step, the response onset and the peak time are defined to build the amplitude and rise time vectors.

\subsection{Data classification}

Analysis of the variance (ANOVA) is a statistical model that provides a statistical test of whether or not the means of several groups are equal. In this case, it generalizes t-test to more than two groups [17].

ANOVA classifier evaluates the value of between-groups variance compared to the average variance within groups. In the case that the between-group variances are identical, mean differences between groups. The ratio of betweengroup variance to within-group variance is the main focus of the ANOVA and the equation is provided in (2). ANOVA performs analysis by comparing the sample dataset and produces a single $p$-value. The significance level that we assume here is 0.05 . If the $P$-value ratio is larger than the expected coincidentally, it results that at least one means is substantially dissimilar. The amount of P-value derives from the cdf of the F-distribution. Thus, to achieve a statistical result the $F$ value calculated from the observed data and the critical value at the a error level of 0.05 should be compared $[28,29]$.

$F=\frac{\text { Between }- \text { Group variance }}{\text { Within }- \text { Group variance }}$

All the statistical results are obtained using the laboratory-developed software based on MATLAB. In this stage, a t-test is applied to compare various stress levels. Besides, the analysis of variance is performed to evaluate the proposed classification results. If the obtained P-value is smaller than 0.05 , then the null hypothesis is rejected and the between-group differences are statistically significant. Therefore, this method could be reliable to discriminate stress levels.

\section{Results and discussion}

In this study, the amplitude and rise time features are employed to classify the stress levels. To investigate the significant difference between the mean of these extracted features, the variance test is used and the results of sixteen sets of experiments of one hand and one foot GSR signals for eight drivers) are assessed. Each experiment consists of two low-stress segments, two moderate-stress segments,
Table 2 Anova table using hand-amplitude feature

\begin{tabular}{llllll}
\hline Variability & SS & df & MS & F & Probe $>F$ \\
\hline Between groups & 0.11547 & 2 & 0.05773 & 16.33 & $9.25472 E-08$ \\
Within groups & 6.94381 & 1964 & 0.00354 & NA & NA \\
Total & 7.05928 & 1966 & NA & NA & NA \\
\hline
\end{tabular}

Table 3 Anova table using hand-time feature

\begin{tabular}{llllll}
\hline Variability & SS & df & MS & F & Probe $>F$ \\
\hline Between groups & $3,832,204$ & 2 & $191,610.2$ & 43.62 & $2.91095 E-19$ \\
Within groups & $8,626,948$ & 1964 & 4392.5 & NA & NA \\
Total & $9,010,168$ & 1966 & NA & NA & NA \\
\hline
\end{tabular}

Table 4 Anova table using foot-amplitude feature

\begin{tabular}{llllll}
\hline Variability & SS & df & MS & F & Probe $>F$ \\
\hline Between groups & 0.03337 & 2 & 0.01669 & 311.17 & Probe $>F$ \\
Within groups & 0.11122 & 2074 & 0.00005 & NA & $6.5672 E-119$ \\
Total & 0.14459 & 2076 & NA & NA & NA \\
\hline
\end{tabular}

Table 5 Anova table using foot-time feature

\begin{tabular}{llllll}
\hline Variability & SS & df & MS & F & Probe $>F$ \\
\hline Between groups & $776,913.9$ & 2 & 388,457 & 150.21 & $1.19363 E-61$ \\
Within groups & $5,363,496$ & 2074 & 2586.1 & NA & NA \\
Total & $6,140,410$ & 2076 & NA & NA & NA
\end{tabular}

and three high-stress segments. Each of the 112 sections is investigated statistically and the corresponding P-value results are identified. The ANOVA tables for hand and foot GSR signals are presented in Tables 2, 3, 4 and 5.

SS indicates the sum of squares of the errors, $\mathrm{df}$ is the degree of freedom and the total degrees of freedom equal to the total number of observations minus one. Moreover, the between-groups degree of freedom equals the number of groups minus one and within groups degree of freedom is the total degree of freedom minus the between groups degree of freedom. Also, MS is the mean squared error and the F-static is the ratio of the mean squared errors. The $P$-value is the probability that the test statistic can take a value greater than the value of the computed test statistics $P(F>0.05)$, the small $P$-value indicates that differences between column means are significant and we can reject $\mathrm{H}_{0}$.

Boxplots illustrate the upper and lower confidence limit of the median for each group. The boxplots of both features considering foot and hand GSR signals are shown in Figs. 6, 7, 8 and 9 . 


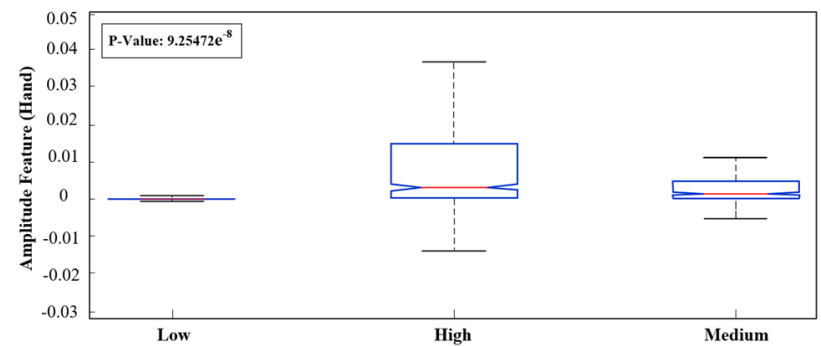

Fig. 6 Classification performance of hand amplitude feature

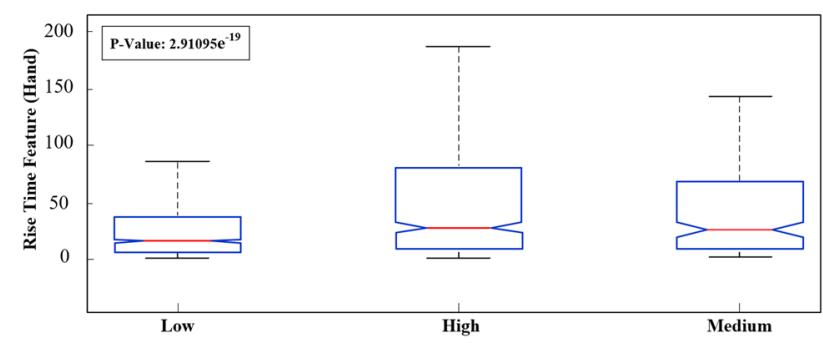

Fig. 7 Classification performance of hand time feature

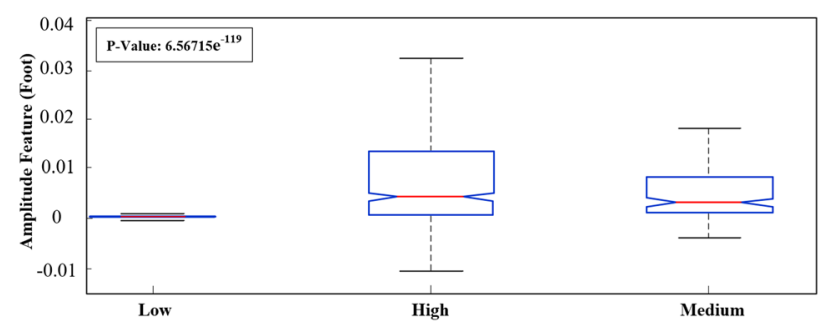

Fig. 8 Classification performance of foot amplitude feature

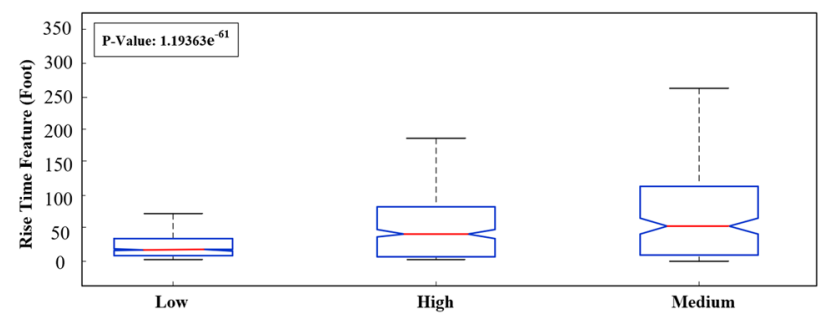

Fig. 9 Classification performance of foot time feature

Calculated between-group P-value results of amplitude and rise time for all eight drivers for both 'foot' and 'hand' signals are shown in Figs. 10, 11, 12, 13. It is worthwhile to mention that $\mathrm{P}$-values more than 0.05 is considered as an error which reduces the precision of classification.

According to the results, the extracted foot GSR amplitude feature provides more precise information about the

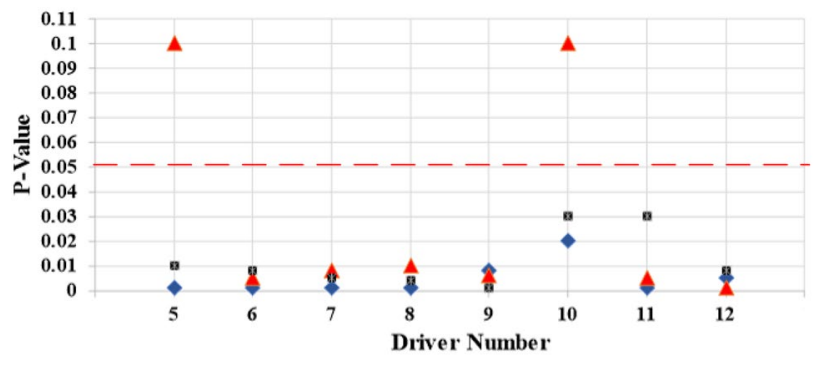

-Low-High $\triangle$ Low-Moderate "Moderate-Hight

Fig. $10 P$-value of hand's amplitude feature

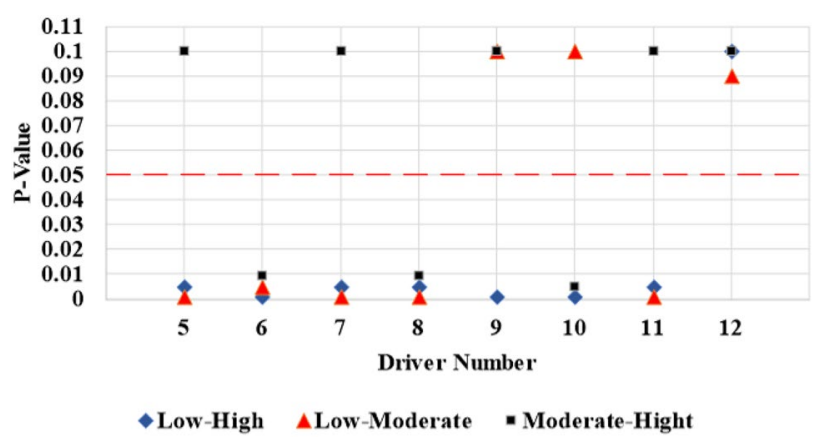

Fig. $11 P$-value of hand's time feature

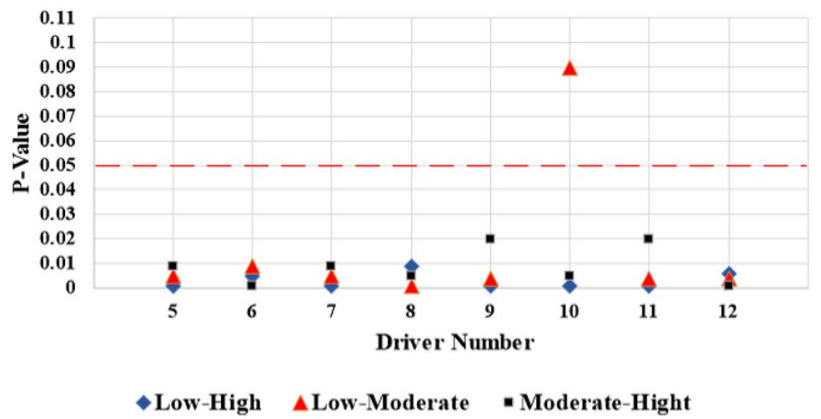

Fig. $12 P$-value of foot's amplitude feature

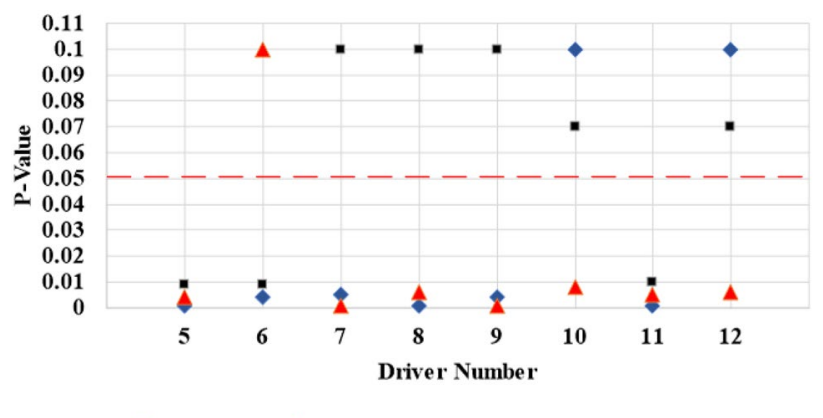

-Low-High ALow-Moderate - Moderate-Hight

Fig. $13 P$-value of foot's time feature

SN Applied Sciences 
Table 6 Discriminant accuracy between different stress levels using amplitude feature of hand and foot signal for 8 drivers

\begin{tabular}{llll}
\hline Stress levels & & \multicolumn{2}{l}{$\begin{array}{l}\text { Between groups discriminant } \\
\text { accuracy }\end{array}$} \\
\cline { 3 - 4 } & & Hand signal & Foot signal \\
\hline Low & High & $100 \%$ & $100 \%$ \\
Low & Med & $75 \%$ & $87.5 \%$ \\
High & Med & $100 \%$ & $100 \%$ \\
Average accuracy & $91.6 \%$ & $95.83 \%$ \\
\hline
\end{tabular}

Table 7 Discriminant accuracy between different stress levels using time feature of hand and foot signal for 8 drivers

\begin{tabular}{llll}
\hline Stress levels & & \multicolumn{2}{l}{$\begin{array}{l}\text { Between groups discriminant } \\
\text { accuracy }\end{array}$} \\
\cline { 3 - 4 } & & Hand signal & Foot signal \\
\hline Low & High & $87.5 \%$ & $100 \%$ \\
Low & Med & $62.5 \%$ & $87.5 \%$ \\
High & Med & $75 \%$ & $100 \%$ \\
Average accuracy & $75 \%$ & $91.6 \%$ \\
\hline
\end{tabular}

drivers' stress level. Besides, $P$-values of all drivers are compared and results show that there is a significant difference between all three levels of stress considering the amplitude feature. On the other hand, recognition between high and moderate stress levels has lower accuracy using the hand signal time feature. Average discrimination accuracy for different signals considering amplitude and time features are presented in Tables 6, 7, respectively.

It is concluded that the amplitude feature is the most informative source to identify stress levels. Moreover, the foot GSR signal presents a high accuracy of stress recognition compared to the hand signal. Using the ANOVA classification model provides us with an average classification rate of $95.83 \%$.

\section{Conclusion}

The focus of this research is on the stress classification based on the GSR signal during the driving task. For this aim, the ANOVA classifier model is employed as a classification tool. The GSR data sets are derived from the MIT Media Lab. It is concluded that the ANOVA model has an excellent performance in classifying three levels of stress based on $P$-value calculations with a $95.83 \%$ rate of accuracy in discriminating three levels. After a thorough study, we can conclude that GSR signals could individually be a reliable data source for stress recognition and classification particularly with the employment of amplitude features of the foot and ANOVA approach. The need for less expensive sensors and less interference with the driver particularly during the driving task endorses the fact that a single signal measurement procedure would be an ideal perspective for future stress detection devices. It must be noted that the acquisition of this data is easier and less prone to hassles compared to other physiological signals. Although the achieved accuracy in this paper is promising and the acquisition of GSR signal is considerably easier compared to other physiological signals, the effect of external temperature in skin conductance which might lead to some errors is undeniable [30]. Since this research is based on previously collected data on the Physionet website, utilizing more precise and comprehensive data would improve the accuracy of classification.

The perspective of future research could be achieving a noncontact stress calculator system which could have hassle-free and cheap implementation along with minimum involvement of external factors and high accuracy. A reliable stress measurement procedure that would allow the driver to participate in the driving task more conveniently could be a future direction.

\section{Compliances with ethical standards}

Conflict of interest The authors have no conflicts of interest to declare that are relevant to the content of this article.

Open Access This article is licensed under a Creative Commons Attribution 4.0 International License, which permits use, sharing, adaptation, distribution and reproduction in any medium or format, as long as you give appropriate credit to the original author(s) and the source, provide a link to the Creative Commons licence, and indicate if changes were made. The images or other third party material in this article are included in the article's Creative Commons licence, unless indicated otherwise in a credit line to the material. If material is not included in the article's Creative Commons licence and your intended use is not permitted by statutory regulation or exceeds the permitted use, you will need to obtain permission directly from the copyright holder. To view a copy of this licence, visit http://creativecommons .org/licenses/by/4.0/.

\section{References}

1. Healey JA, Picard RW (2005) Detecting stress during real-world driving tasks using physiological sensors. IEEE Trans Intell Transp Syst 6(2):156-166

2. Lohani M, Payne BR, Strayer DL (2019) A Review of Psychophysiological Measures to Assess Cognitive States in Real-World Driving. Front Hum Neurosci 13:1-27

3. Bitkina OV, Kim J, Park J, Park J, Kim HK (2019) Identifying Traffic Context Using Driving Stress: A Longitudinal Preliminary Case Study. Sensors 19(9):2152

4. Martinez R, Irigoyen E, Arruti A, Martin Jl, Muguerza J (2017) A real-time stress classification system based on arousal analysis 
of the nervous system by an F-state machine. Comput Methods Programs Biomed 148:81-90

5. Sharma N, Gedeon T (2012) Objective measures, sensors and computational techniques for stress recognition and classification: A survey. Comput Methods Programs Biomed 108(3):1287-1301

6. G. Rigas, C. D. Katsis, P. Bougia, and D. I. Fotiadis, "A reasoningbased framework for car driver's stress prediction," 2008 Mediterr. Conf. Control Autom.-Conf. Proceedings, MED'08, pp. 627632, 2008.

7. Zhai J, Barreto A (2006) Stress detection in computer users through non-invasive monitoring of physiological signals. Biomed Sci Instrum 42:495-500

8. Angus F, Zhai J, Barreto A (2005) Front-end analog pre-processing for real time psychophysiological stress measurements, WMSCI 2005-9th World Multi-Conference Syst. Cybern. Informatics, Proc., 5, 218-221

9. Wang JS, Lin CW, Yang YTC (2013) A k-nearest-neighbor classifier with heart rate variability feature-based transformation algorithm for driving stress recognition. Neurocomputing 116:136-143

10. de Vries GJJ, Pauws SC, Biehl M (2015) Insightful stress detection from physiology modalities using Learning Vector Quantization. Neurocomputing 151(P2):873-882

11. Vicente J, Laguna P, Bartra A, Bailón R (2016) Drowsiness detection using heart rate variability. Med Biol Eng Comput 54(6):927-937

12. Singh M, Bin Queyam A (2013) Stress detection in automobile drivers using physiological parameters: a review. Int. J. Electron. Eng 2:1-5

13. Shi Y, Ruiz N, Taib R, Choi E, Chen F (2007) Galvanic skin response (GSR) as an index of cognitive load. In: $\mathrm{CHI}^{\prime} 07$ extended abstracts on Human factors in computing systems, pp 2651-2656

14. Rowe DW, Sibert J, Irwin D (1998) Heart rate variability: indicator of user state as an aid to human-computer interaction, Conf. Hum. Factors Comput. Syst.-Proc., no. February, pp. 480-487

15. Zangróniz R, Martínez-Rodrigo A, Pastor JM, López MT, Fernández-Caballero A (2017) Electrodermal activity sensor for classification of calm/distress condition. Sensors (Switzerland) 17(10):1-14

16. Tang TB, Yeo LW, Lau DJH (2014) Activity awareness can improve continuous stress detection in galvanic skin response. Proc. IEEE Sensors 2014:1980-1983

17. Bharathi A, Natarajan AM (2010) Cancer classification of bioinformatics datausing ANOVA. Int J Comput Theory Eng 2(3):369-373

18. Ogorevc J, Geršak G, Novak D, Drnovšek J (2013) Metrological evaluation of skin conductance measurements. Meas J Int Meas Confed 46(9):2993-3001

19. Schmidt M, Penner D, Burkl A, Stojanovic R, Schümann T, Beckerle $P$ (2016) Implementation and evaluation of a low-cost and compact electrodermal activity measurement system. Meas $J$ Int Meas Confed 92:96-102

20. Schmidt E, Decke R, Rasshofer R (2016) Correlation between subjective driver state measures and psychophysiological and vehicular data in simulated driving. IEEE Intell. Veh. Symp. Proc 2016:1380-1385

21. Lazzeri N, Mazzei $D$, Greco A, Rotesi A, Lanatà A, De Rossi DE (2015) Can a humanoid face be expressive? A psychophysiological investigation. Front Bioeng Biotechnol 3:64

22. Katsis CD, Goletsis Y, Rigas G, Fotiadis DI (2011) A wearable system for the affective monitoring of car racing drivers during simulated conditions. Transp Res Part C Emerg Technol 19(3):541-551

23. Bianco S, Napoletano P, Schettini R (2019) Multimodal car driver stress recognition. In: Proceedings of the 13th EAI International Conference on Pervasive Computing Technologies for Healthcare, pp 302-307

24. Alić B, Sejdinović D, Gurbeta L, Badnjevic A (2016) Classification of stress recognition using artificial neural network, 5th Mediterr. Conf. Embed. Comput., pp. 297-300

25. Jimenez-Limas MA, Ramirez-Fuentes CA, Tovar-Corona B, GarayJimenez LI (2018) Feature selection for stress level classification into a physiologycal signals set, 15th Int. Conf. Electr. Eng. Comput. Sci. Autom. Control, no. April 2019

26. Nithya B, llango V (2019) Evaluation of machine learning based optimized feature selection approaches and classification methods for cervical cancer prediction. SN Appl Sci 1(6):1-16

27. Schmidt S, Walach $H$ (2000) Electrodermal activity (EDA) - Stateof-the-art measurement and techniques for parapsychological purposes. J Parapsychol 64(2):139-163

28. Wagner J, Kim J, André E (2005) From physiological signals to emotions: implementing and comparing selected methods for feature extraction and classification. IEEE Int. Conf. Multimed Expo, ICME 2005 2005:940-943

29. Panure T, Sonawani S (2019) Stress detection using smartphone and wearable devices: a review. Asian J Converg Technol 05(01):1-4

30. Mane R, Batule B, Lomte N, Gundecha AD (2020) International conference on communication and information processing available on : SSRN RF Energy Harvesting using Efficient Power Management System

Publisher's Note Springer Nature remains neutral with regard to jurisdictional claims in published maps and institutional affiliations. 